\title{
Methodology for assessing the grain mass thermal effusivity in a metal silo under the influence of active ventilation
}

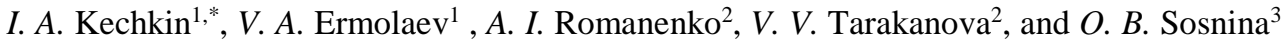 \\ ${ }^{1}$ Department of Commodity Science and Expertise, Plekhanov Russian University of Economics, 117997 Moscow, Russia \\ ${ }^{2}$ K.G. Razumovsky Moscow State University of technologies and management (the First Cossack University), 109004 Moscow, Russia \\ ${ }^{3}$ Department of Technology and Organization of Food Production, Faculty of Business, Novosibirsk State Technical University (NSTU), \\ 630073 Novosibirsk, Russia
}

\begin{abstract}
The purpose of studying the thermal effusivity of the grain mass in a metal silo is to obtain a dynamic mathematical model of the processes of layered changes in the temperature of the grain mass under the influence of an external factor. Such an external factor is the supply of cooling air through the air distribution grid of the bottom of the silo with the active ventilation of grain in a metal silo. The created mathematical and parametric models allow simulating the moisture transfer process using active air ventilation systems inside metal silos during storage of wheat grain. There have been developed some ways to improve the conditions for ensuring the quality preservation of grain during its long-term storage in a metal silo. It is necessary to load such a silo with grain completely, preferably up to the top, leaving free supergrannial space as small as possible. Another solution is thermal insulation of the surfaces of the silo. The task of ensuring the safety of wheat food grain can be accomplished not only on the basis of existing regulatory rules, but also by taking into account the results of new research. The main method of storage in metal containers, according to the instructions and safety regulations is the storage of grain in a dry and purified state. It is known that not only humidity and purity, but also the temperature of the grain put into storage affect the storage period.
\end{abstract}

\section{Introduction}

Reduction of the wheat grain losses during its storage period on a national scale potentially gives results similar to an increase in bulk yield. The task of ensuring the safety of wheat food grain can be accomplished not only on the basis of existing regulatory rules, but also by taking into account the results of new research.

The main method of storage in metal containers, according to the instructions and safety regulations is the storage of grain in a dry and purified state. It is known that not only humidity and purity, but also the temperature of the grain put into storage affect the storage period. The lower the temperature, the longer the storage time becomes.

Metal silo of large capacity intended for grain storage are equipped with two installations of influence on the air space inside the silo: active ventilation of the grain mass and ventilation of the space above the grain under the roof of the silo. Active ventilation systems affect the grain mass with an upward flow of atmospheric air. This system is used to cool the grain mass in order to increase the shelf life, to prevent or eliminate self-heating in it. The installation for ventilation of the over-grain space is used to remove condensate formed during grain storage and settled on the silo structures.
The most unfavorable storage conditions inside a metal silo develop in the upper part of the bulk-grain. If the relative humidity of the air in the silo is higher than the relative humidity of the outside air, it is recommended to provide ventilation of the grain space.

At low air filtration rates, moisture desorbed from the grain settles on the surface of the grain mass, which leads to its humidification.

In order to avoid moisture sedimentation on the surface of the grain mass, it is necessary to ventilate it at filtration rates ensuring the removal of moisture outside the silo.

The purpose of the developed mathematical model is to provide a tool for the calculation of the trajectory of the grain temperature changes in along the layers of the grain mass during an active ventilation of the grain, including changes happen in conditions of variable values of ambient temperatures and with intermittent implementations of active ventilation mode [1].

Due to the pronounced spatial distribution of the thermal object - the grain mass in the silo, the dynamic mathematical model of such an object in the general case should be constructed based on partial differential equations or their grid-based solutions. However, such models are complex both in their parametric identification and in their subsequent application. 


\section{Materials and method}

On the ground of the fact that during active ventilation, heat propagation occurs mainly along the vertical coordinate of the silo, it was decided to simplify the task and to adopt an approach, according to which dynamic modeling is carried out for a number of conjugated objects with lumped parameters. As a series of such objects, the grain mass layers selected along the vertical coordinate of the silo are taken, in each of the layers the output parameter (temperature) is considered uniform within this layer [2].

\section{Results}

The proposed formulation shows that dynamic modeling of a distributed object can be reduced to parametric identification of conjugate aperiodic links, supplemented by links of pure delay [3]. The block diagram of the model in parallel expansion is presented in Fig. 1.

Another option for decomposing elements in a model is a sequential model. Such model is presented in Fig. 2. Here, each subsequent layer receives an input signal from the previous layer.

The subsequent application of such simplified models can be carried out separately for each of the grain mass layers selected according to the height of the silo [4].

The very application of the model is to obtain solutions of the corresponding differential equations in the time domain.
In practical terms, the height of each of these elementary layers of the grain mass is proposed to be selected on the bases of the corresponding distances between the temperature sensors in the standardly used thermal suspensions installed in the silo. In this case, it is advisable to carry out the parametric identification of the models on the ground of data on temperature changes obtained during the active ventilation of grain in a silo in the mode of its normal operation [5].

The indicated parametric identification can be carried out both by analytical methods and graphical ones [6]. One of the simplest graphical methods is the tangent method. The essence of this method is the graphical construction of a tangent line to the line of transition process of the output quantity $(\mathrm{Y})$ - grain temperature that is changing under the influence of the input quantity (X) - active air ventilation temperature. The time interval obtained at the intersection of the tangent line with the steady-state value of the output value is the time constant (T) of the dynamic link. Accordingly, the dimension of this parameter is a unit of time [7]. The second parameter - the transmission coefficient of the dynamic link $(\mathrm{K})$ has a dimensionless form and is defined as the ratio of the input and output quantities in its steady-state value [8]. The parameter of pure time delay $(\tau)$ is defined as the time interval at the initial stage of the transition process during which the output value has not undergone significant changes after the start of the input stimulus (Fig. 3).

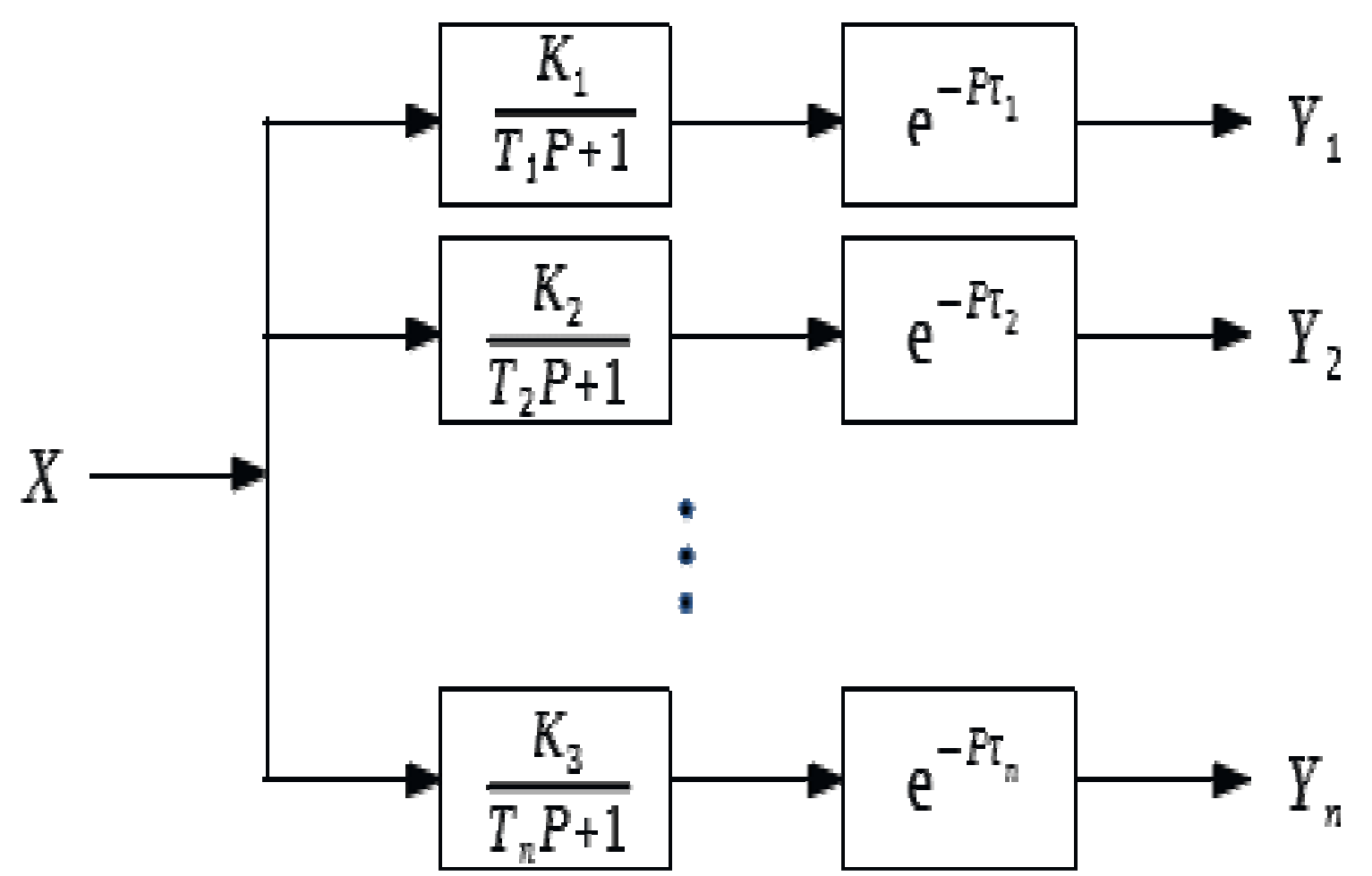

Fig. 1. Block diagram of a parallelized mathematical model for cooling grain in a silo, where $\mathrm{X}$ is the input variable; $\mathrm{Y} 1$ - Yn are output variables by layers; $\mathrm{K} 1-\mathrm{Kn}$ are transfer coefficients; $\mathrm{T} 1-\mathrm{Tn}$ are time constants; $\mathrm{P}$ is the Laplace operator 


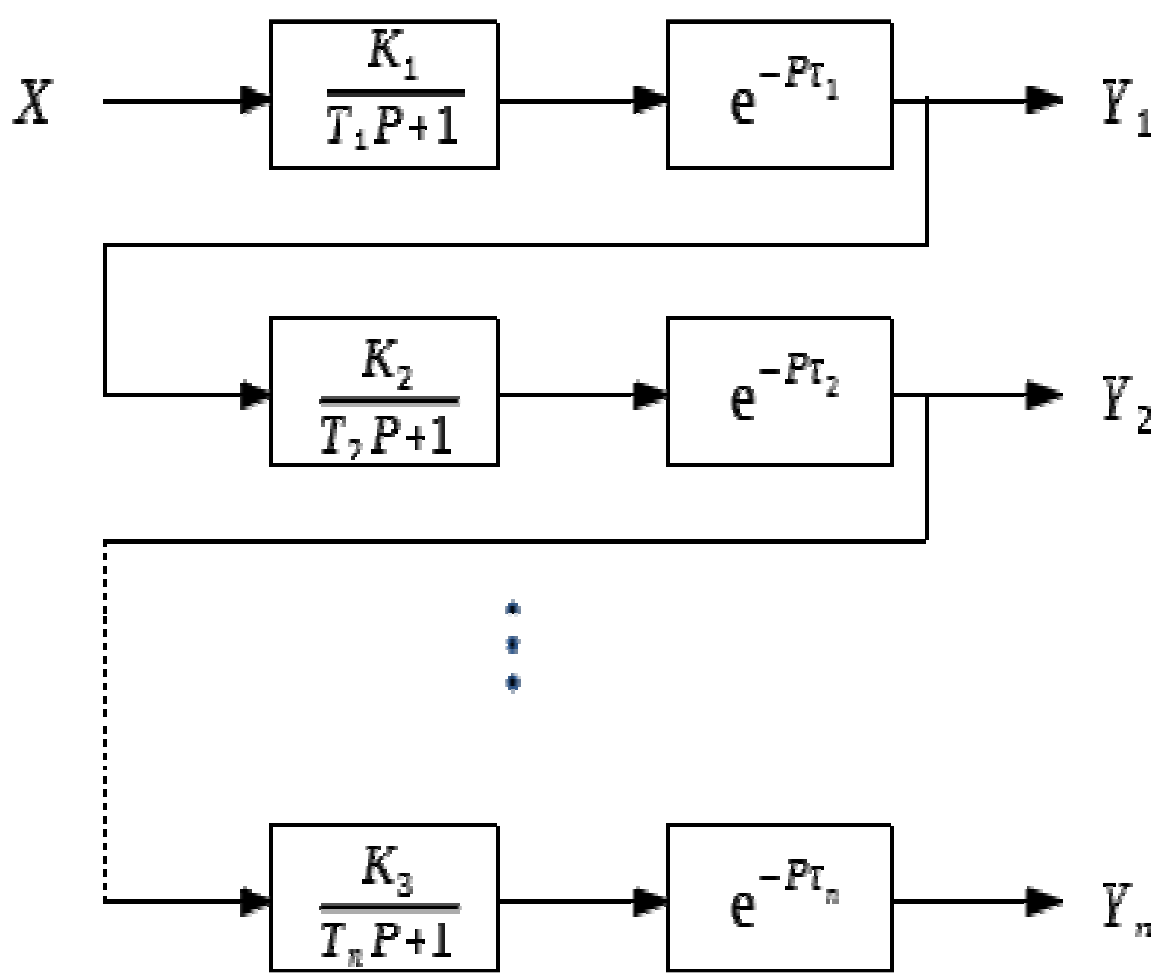

Fig. 2. Block diagram of a mathematical model for cooling grain in a silo in sequential decomposition

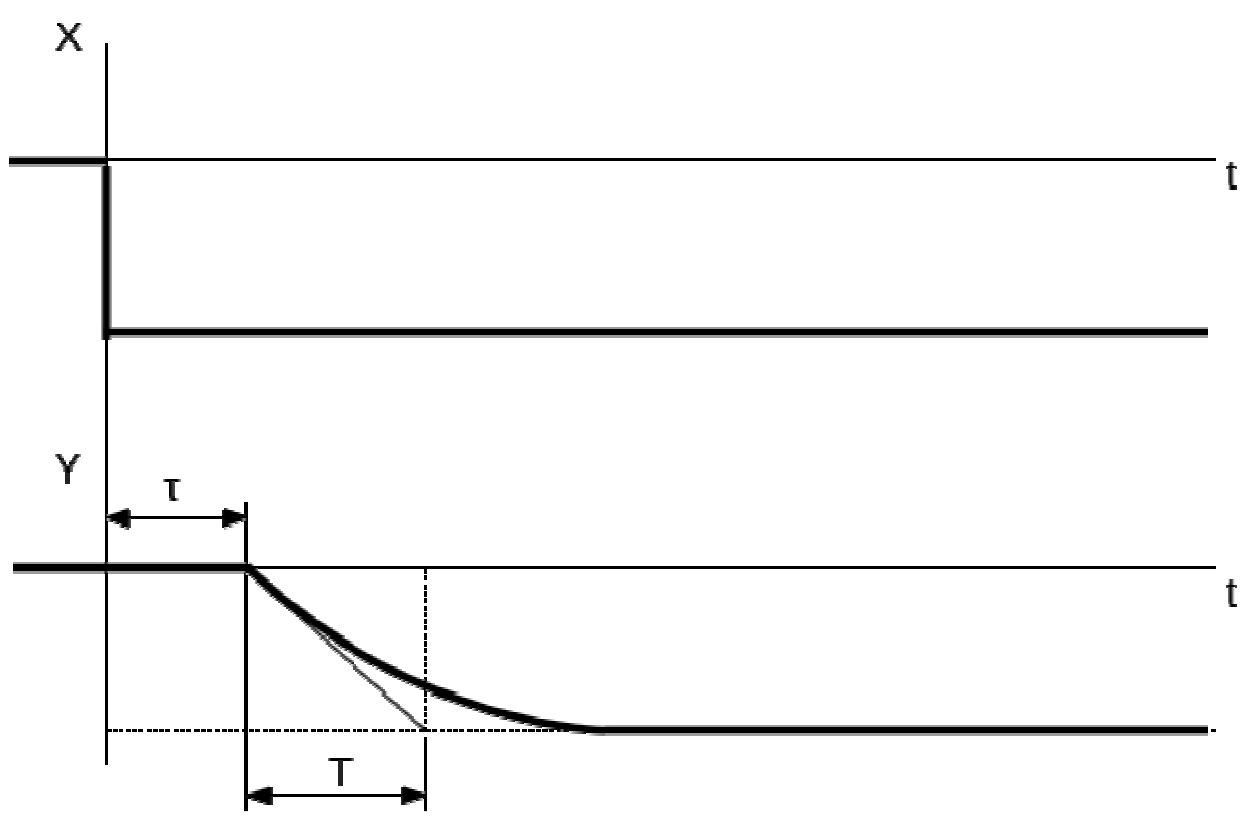

Fig. 3. Parametric identification of a model by the tangent method

\section{Methodology for assessing critical changes in boundary conditions during grain storage in a metal silo}

Preliminary results of the study revealed a significant influence of the state of the under-roof space of a metal silo on the processes of heat and mass transfer into the stored grain mass and the possible concentration of the transferred moisture in critical zones of this grain mass [9].

In this regard, a series of experiments it has been methodically prepared, allowing for instrumental assessment of the moisture condensation effect on the inner surfaces of the roof and unfilled walls of a metal silo in the mode of its normal operation [10].

It should be emphasized that moisture condensation inside the silo is possible only on surfaces that are not in contact with grain. The air of intergranular space behaves in a completely different way than the air above the grain. And the reason for this is that between the grain and the air of the intergranular space, the processes of mass transfer balancing proceed much more intensively than between the surface of the grain mass and the air of the supergrannial space. 
It is the process of condensation of the moisture contained in the supergrannial air, on the internal unfilled surfaces of the metal silo that essentially presents the converter of the bound moisture of the grain into unbound condensed moisture, which ultimately returns in this free form to the grain surface and creates additional conditions for its microbiological damage [11].

An important feature of the process is that such moisture condensation on the internal surfaces of a metal silo, which are not in contact with grain, is possible under quite certain conditions [12]. Such conditions are the relatively high temperature of the grain stored inside the metal silo and the relatively low ambient temperature outside. This situation regularly occurs in the autumnwinter period, when the grain has not been cooled yet due to its still high average ambient temperature or the lack of production capacity to carry out such an operation, but the ambient temperature regularly falls below the levels leading to moisture condensation on internal non-contacting with grain surfaces of a metal silo [13].

The assessment of conditions for moisture condensation formation must be determined both by calculation - in an interactive numerical modeling system, and by the method of obtaining experimental data on the state of the inner surface of the under-roof space of a metal silo under conditions of its normal operation [14].

\section{Conclusion}

The created mathematical and parametric models allow simulating the moisture transfer process using active air ventilation systems inside metal silos during storage of wheat grain.

Some ways have been developed to improve the conditions for ensuring the quality preservation of grain during its long-term storage in a metal silo:

1. It is necessary to load such a silo with grain completely, preferably up to the top, leaving free supergrannial space as small as possible. This helps to minimize the area of the inner surfaces of the metal silo, on which moisture condensation is possible to form, and its subsequent contact with the grain. It should be borne in mind that not all the designs of metal silos allow loading grain up to the top.

2. Another effective method of reducing the possible condensation of moisture formation on the inner surface of the roof is the proper organization of its convective ventilation. In house-building technologies, such a problem is well known and has proven solutions that can be applied very well to provide ventilation of the underroof space of a metal silo. The essence of such decisions is that in the under-roof space the directional movement of convective upward flows of external air is specially organized. Such free air convection in the under-roof space can be achieved by installing a set of special air supply and air discharge aerators, moreover, air discharge aerators must be installed at the top levels of the roof, and air supplying aerators are closer to the lowest roof cut. For some roof constructions manufacturers declare an annular gap along the lower perimeter of the roof - it could be considered as a distributed supply aerator. Due to the location of the discharge and air supplying aerators spaced apart in height and their corresponding distribution over the entire roof area, the air flow could be organized in direct contact with the inner surface of the roof, which can be defined as "draft" and which is less prone to condensation in its parameters on this inner surface. Based on the considered positions, then on the outer surface of the roof should be installed a calculated set of aerators - discharge and air supplying.

However, this is a task for manufacturers of silos such installation cannot be fully implemented by an operating organization. Manufacturers of silos do not pay enough attention to this problem. The standard air distribution devices installed on the roof of the silo should essentially solve the problem of air inlet / outlet from the silo / into the silo during loading / unloading of grain, as well as with active air ventilation of grain in the silo. Numerous materials at our disposal confirm that the practiced arrangement of such air-distributing devices on the silo roof does not provide full convective ventilation of the inner surfaces of the silo roof and, accordingly, does not help to reduce the risks of moistening grain with condensation moisture.

3. Another passive technique that can reduce the risks of condensation moisture is the thermal insulation of the surfaces. Moreover, it is first of all advisable to carry out such thermal insulation precisely on the roof of the silo. Thermal insulation of the walls of the silo will give significantly less economic effect due to significantly higher costs (due to the larger area) and lower technological efficiency. The indicated technique also belongs to the category of recommended design solutions for silo manufacturers.

\section{References}

1. V. Vassiliev, F. Magni, S. Chernishev, V. Kostege, Impact of the $3 d$ flow effects on the silo combustor thermal state, Proc. of the ASME Turbo Expo Cep., 881-890 (2012)

2. M.B. Toffolo, E. Boaretto, M.A.S. Martin, D.M. Master, Microarchaeology of a grain silo: insights into stratigraphy, chronology and food storage at late bronze age ashkelton, Israel J. of Archaeolog. Sci., 177-188 (2018)

3. M.R. Bonner, M.C.R. Alavanja, Pesticides, human health, and food security, Food and Energy Secur., 6(3), 89-93 (2017)

4. W.J. Davies, J.M. Ribaut, Stress resilience in crop plants: Strategic thinking to address local food production problems, Food and Energy Secur. 6(1), 12-18 (2017)

5. C. Pollock, Sustainable farming: Chasing a mirage? Food and Energy Secur., 5(4), 205-209 (2016)

6. B.H. Cervelin, D. Conti, M.A. Diniz-Ehrhardt, J.M. Martínez, A computer model for particle-like 
simulation in broiler houses, Computers and Electron. in Agricult., 1-14 (2017)

7. M.J. Oates, A. Ruiz-Canales, M. Ferrández-Villena, A.F. López, A low cost sunlight analyser and data logger measuring radiation, Computers and Electron. in Agricult., 38-48 (2017)

8. C. Gilmore, M. Asefi, J. LoVetri, J. Paliwal, Industrial scale electromagnetic grain bin monitoring, Computers and Electron. in Agricult., 210-220 (2017)

9. P.J. García Nieto, E. García-Gonzalo, J. Bové, M. Duran-Ros, J. Puig-Bargués, Modeling pressure drop produced by different filtering media in microirrigation sand filters using the hybrid $A B C$ MARS-based approach, MLP neural network and M5 model tree, Computers and Electron. in Agricult., 65-74 (2017)

10. Y. Huang, C. Li, Real-time monitoring system for paddy environmental information based on DC powerline communication technology, Computers and Electron. in Agricult., 51-62 (2017)
11. G.D. Stone, D. Glover, Disembedding grain: Golden Rice, the Green Revolution, and heirloom seeds in the Philippines, Agricult. and Human Values, 87-102 (2017)

12. M. Bhatta, V. Belamkar, P.S. Baenziger, A. Morgounov, Genome-wide association study reveals novel genomic regions for grain yield and yield-related traits in drought-stressed synthetic hexaploid wheat, Int. J. of Molecular Sci., 19(10) (2018)

13. I. Kechkin, V. Ermolaev, A. Romanenko, M. Ivanov, E. Gurkovskaya, Dependence of fat acidity value on wheat grain storage conditions, Int. Conf. on Food Industry, Economy and Security, pp. 34-39 (2020)

14. I. Kechkin, V. Ermolaev, A. Romanenko, V. Tarakanova, K. Buzetti, Management of air flows inside steel silo during grain storage, Int. Conf. on Food Industry, Economy and Security, pp. 22-25 (2020) 